\title{
Are correctional facilities amplifying the epidemic of community-acquired methicillin-resistant Staphylococcus aureus?
}

\section{Justin T. Okano and Sally Blower}

In their recent Review (Waves of resistance: Staphylococcus aureus in the antibiotic era. Nature Rev. Microbiol. 7, 629-641 (2009)) ${ }^{1}$ Chambers and DeLeo described the emergence, over the past decade, of an epidemic of community-acquired methicillin-resistant Staphylococcus aureus (CA-MRSA). As they discussed, it is unclear what factors are driving this epidemic of resistance. We propose that, in some areas, correctional facilities may be worsening the epidemic of CA-MRSA by acting as 'amplification zones'.
The United States has the highest incarceration rate in the world. In 2007, 1 out of every 31 adults in the United States (that is, over 7.3 million people) were incarcerated, on probation or on parole ${ }^{2}$. Over the past few years large outbreaks of CA-MRSA have occurred in correctional facilities in California, Texas, Missouri, Georgia and Mississippi $^{3-7}$, and recent incarceration has been identified as an important risk factor for acquiring CA-MRSA ${ }^{8,9}$. Taken together, these facts indicate that correctional facilities may have the potential to act as amplification zones for CA-MRSA.

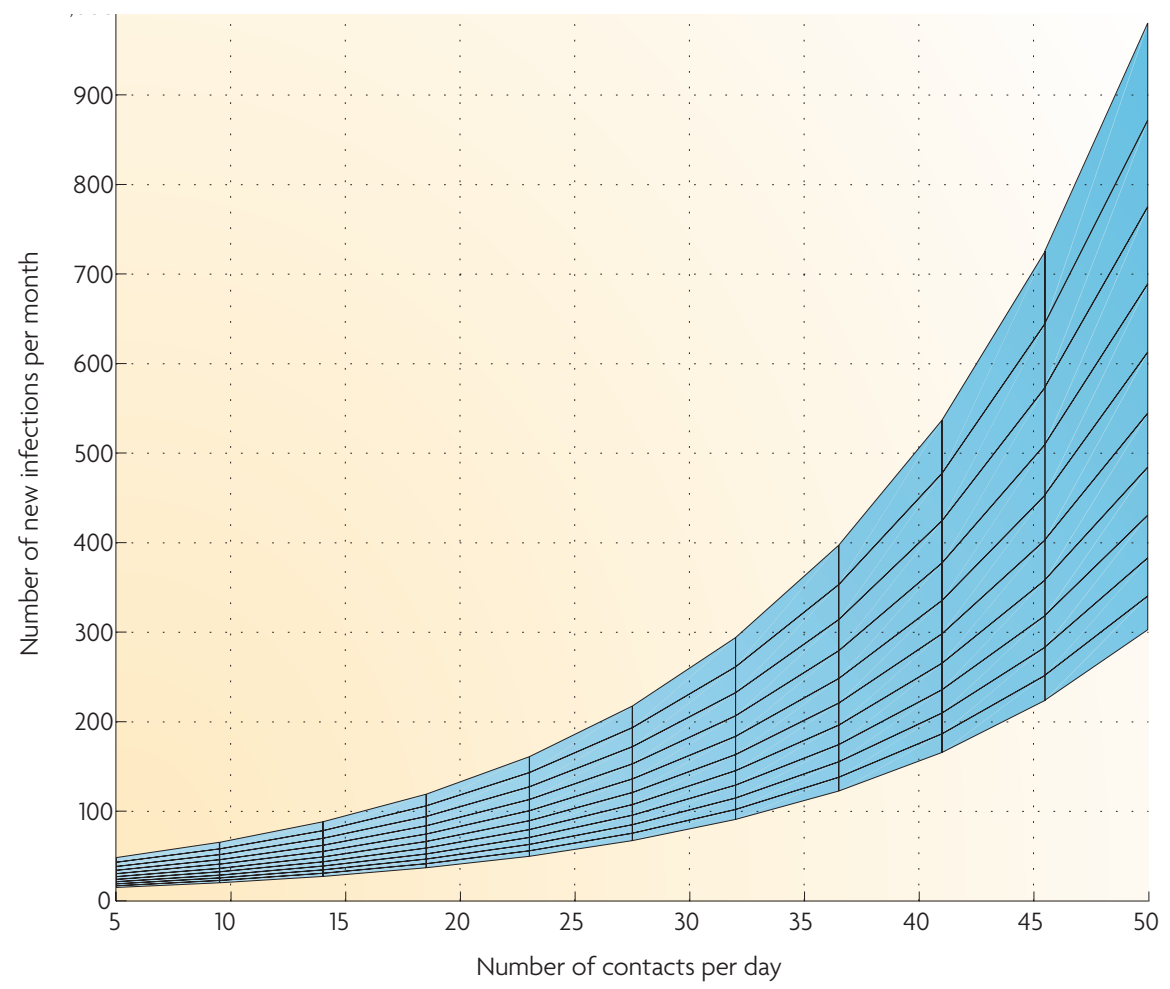

Figure 1 Effect of crowding on the spread of CA-MRSA in jail. The bottom curve represents the number of male inmates in the Los Angeles County Jail (LACJ) infected per month assuming that only one individual infected with community-acquired methicillin-resistant Staphylococcus aureus (CA-MRSA) enters the jail per month from the community. The top curve represents the number of male inmates in the LACJ infected per month assuming that 60 individuals infected with CA-MRSA enter the jail per month from the community. The $x$ axis shows the degree of crowding in the LACJ, as expressed by the number of contacts an inmate has with other inmates per day. This graph was generated using the mathematical model described in REF. 10.
The Los Angeles County Jail (LACJ) is experiencing one of the largest outbreaks of CA-MRSA of any correctional facility ${ }^{10}$. It is also the largest jail in the world, housing $\sim 165,000$ inmates per year, with $\sim 20,000$ inmates incarcerated at any one time. Ten to twelve thousand male inmates are released from this facility per month. The CA-MRSA outbreak in the LACJ began in 2001 and initially grew exponentially ${ }^{10}$; to date over 9,000 infections have been reported. We have previously developed a mathematical model for tracking the transmission dynamics of CA-MRSA in the $\mathrm{LACJ}^{10}$. Our model can be used to assess the potential role of the LACJ as an amplification zone.

FIGURE 1 shows results from our model of the potential effect of the CA-MRSA epidemic in Los Angeles County on the LACJ outbreak. The bottom curve represents within-jail incidence of infection assuming that only one of the men entering the jail per month is infected with CA-MRSA. The top curve represents within-jail incidence of infection assuming that 60 of the men entering the jail per month are infected. Within-jail incidence will be relatively low if inmates have only a few daily contacts with other inmates (FIG. 1). Under these conditions the magnitude of the outbreak will be relatively unaffected by the rate of inflow of infected individuals from the community (FIG. 1). However, if the jail is crowded and daily contacts with other inmates are frequent, a high rate of inflow of infected individuals could result in a very high incidence of infection in the jail (FIG. 1). Consequently, several thousands of the inmates who are released into the surrounding community per month could be infected with CA-MRSA (FIG. 2). This large influx of infected individuals is likely to increase CA-MRSA transmission in the community. Therefore, our modelling shows that a crowded correctional facility has the potential to act as an amplification zone for CA-MRSA.

The prevalence of CA-MRSA is increasing in Los Angeles County. As the LACJ is very crowded and the current outbreak is extremely large, we suggest that the LACJ may be acting as a significant amplification zone. Other correctional facilities in the United States may also be amplifying the spread of CA-MRSA and contributing to the rising epidemic of resistance.

Justin T. Okano and Sally Blower are at the Center for Biomedical Modeling, Semel Institute of Neuroscience \& Human Behaviour, David Geffen School of Medicine at UCLA, 1100 Glendon Avenue PH2, Los Angeles, California 90024, USA. Correspondence to S.B. e-mail:sblower@mednet.ucla.edu 


\section{CORRESPONDENCE}

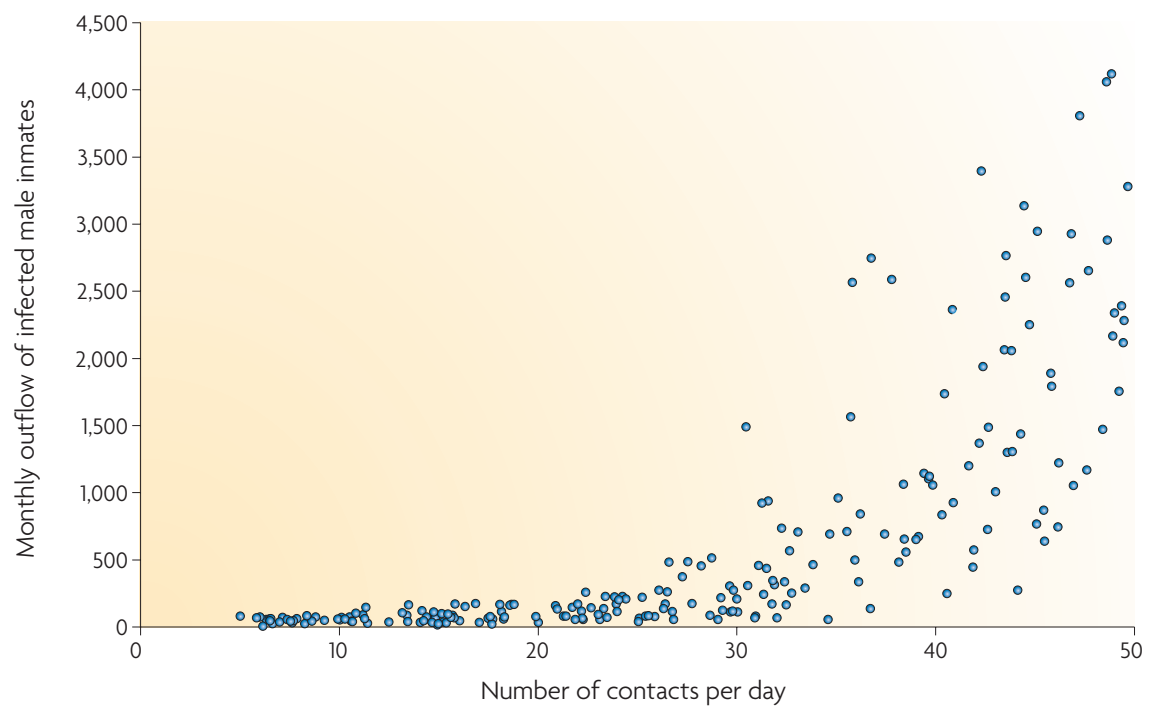

Figure 2 | Effect of crowding on the level of CA-MRSA in released prisoners. The relationship between the number of male inmates infected with community-acquired methicillin-resistant Staphylococcus aureus (CA-MRSA) who are released from the Los Angeles County Jail per month and the degree of crowding in the jail (as expressed by the number of contacts an inmate has with other inmates per day). This graph was generated using the mathematical model described in REF. 10.

1. Chambers, H. F. ¿ Deleo, F. R. Waves of resistance: Staphylococcus aureus in the antibiotic era. Nature Rev. Microbiol. 7, 629-641 (2009).

2. US Department of Justice. Correction statistics. Bureau of Justice Statistics [online], http://www.ojp. usdoj.gov/bjs/correct.htm (2009).

3. Turabelidze, G. et al. Personal hygiene and methicillinresistant Staphylococcus aureus infection. Emerg. Infect. Dis. 12, 422-427 (2006).

4. Baillargeon, J., Kelley, M. F., Leach, C. T., Baillargeon, G. ¿ Pollock, B. H. Methicillin-resistant Staphylococcus aureus infection in the Texas prison system. Clin. Infect. Dis. 38, e92-e95 (2004).

5. CDC. Methicillin-resistant Staphylococcus aureus infections in correctional facilities - Georgia, California, and Texas, 2001-2003. Morb. Mortal. Wkly Rep. 52, 992-996 (2003).

6 CDC. Methicillin-resistant Staphylococcus aureus skin or soft tissue infections in a state prison Mississippi, 2000. Morb. Mortal. Wkly Rep. 50, 919-922 (2001)
7. CDC. Outbreaks of community-associated methicillinresistant Staphylococcus aureus skin infections - Los Angeles County, California, 2002-2003. Morb. Mortal. Wkly Rep. 52, 88 (2003)

8. Hota, B. et al. Community-associated methicillinresistant Staphylococcus aureus skin and soft tissue infections at a public hospital: do public housing and incarceration amplify transmission? Arch Intern. Med. 167, 1026-1033 (2007)

9. Jacobus, C. H. et al. Prevalence and demographics of methicillin resistant Staphylococcus aureus in culturable skin and soft tissue infections in an urban emergency department. BMC Emerg. Med. 7, 19 (2007).

10. Kajita, E., Okano, J. T., Bodine, E. N., Layne, S. P. \& Blower, S. Modelling an outbreak of an emerging pathogen. Nature Rev. Microbiol. 5, 700-709 (2007).

Competing interests statement

The authors declare no competing financial interests. 